10 Edmonds P, Karlsen S, Khan S, et al. A comparison of the palliative care needs of patients dying from chronic respiratory diseases and lung cancer. Palliat Med 2001; 15: 287-295.

11 Elkington $\mathrm{H}$, White $\mathrm{P}$, Addington-Hall J, et al. The healthcare needs of chronic obstructive pulmonary disease patients in the last year of life. Palliat Med 2005; 19: 485-491.

12 Claessens MT, Lynn J, Zhong Z, et al. Dying with lung cancer or chronic obstructive pulmonary disease: insights from SUPPORT. Study to Understand Prognoses and Preferences for Outcomes and Risks of Treatments. J Am Geriatr Soc 2000; 48: Suppl., S146-S153.

13 Simon ST, Altfelder N, Alt-Epping B, et al. Is breathlessness what the professional says it is? Analysis of patient and professionals' assessments from a German nationwide register. Support Care Cancer 2014; 22: 1825-1832.

14 Higginson IJ, Bausewein C, Reilly CC, et al. An integrated palliative and respiratory care service for patients with advanced disease and refractory breathlessness: a randomised controlled trial. Lancet Respir Med 2014; 2: 979-987.

15 Abernethy AP, Currow DC, Frith P, et al. Randomised, double blind, placebo controlled crossover trial of sustained release morphine for the management of refractory dyspnoea. BMJ 2003; 327: 523-528.

16 Ekstrom MP, Bornefalk-Hermansson A, Abernethy AP, et al. Safety of benzodiazepines and opioids in very severe respiratory disease: national prospective study. BMJ 2014; 348: g445.

\title{
Effects of ageing and smoking on pulmonary computed tomography scans using parametric response mapping
}

To the Editor:

Chronic obstructive pulmonary disease (COPD) is an obstructive lung disease often caused by cigarette smoke, and characterised by inflammation and abnormalities of the large and small airways (i.e. those with an internal diameter $<2 \mathrm{~mm}$ ), as well as by alveolar destruction (emphysema). Recent evidence suggests that small airway disease precedes emphysema [1] and, therefore, it may be useful to identify the presence and extent of small airway disease and emphysema in early COPD, or preferably, even before the onset of disease.

Parametric response mapping (PRM) is a novel technique to analyse pulmonary computed tomography (CT) scans in order to quantify the extent of small airway disease $\left(\mathrm{PRM}^{\mathrm{fSAD}}\right)$, emphysema $\left(\mathrm{PRM}^{\mathrm{Emph}}\right.$ ) and parenchymal disease $\left(\mathrm{PRM}^{\mathrm{PD}}\right)$, the latter reflecting increased attenuation of normal lung parenchyma $[2,3]$. We aimed to evaluate the PRM technique in a cohort of well-characterised, respiratory-healthy subjects with a wide age range. As smoking and ageing are both risk factors in the development of COPD [4], we hypothesised that 1) an older age is associated with more $\mathrm{PRM}^{\mathrm{fSAD}}, \mathrm{PRM}^{\mathrm{Emph}}$ and $\mathrm{PRM}^{\mathrm{PD}}$, and 2) current smoking is associated with more $\mathrm{PRM}^{\mathrm{fSAD}}, \mathrm{PRM}^{\mathrm{Emph}}$ and $\mathrm{PRM}^{\mathrm{PD}}$. Finally, we investigated the association between PRM measurements and pulmonary function measurements.

We selected current smokers and never-smokers older than 18 years, without respiratory symptoms and with no history of respiratory diseases. In addition, they had normal pulmonary function, defined as a post-bronchodilator forced expiratory volume in $1 \mathrm{~s}(\mathrm{FEV} 1) /$ forced vital capacity (FVC) ratio above the lower limit of normal, no bronchial hyperresponsiveness and reversibility of FEV 1 to salbutamol $<10 \%$ of the predicted value.

Spirometry (FEV1, FVC, FEV1/FVC and forced expiratory flow at 25-75\% of FVC (FEF25-75\%)), body plethysmography (residual volume (RV), total lung capacity (TLC) and RV/TLC) and methacholine provocation tests were performed according to international guidelines $[5,6]$. Transfer factor of the lung for carbon monoxide corrected from haemoglobin (TLCOc) adjusted for alveolar volume (VA) was measured using the single breath-holding technique, and small airway resistance (resistance at $5 \mathrm{~Hz}(R 5)$ minus resistance at $\left.20 \mathrm{~Hz}\left(R_{20}\right)\right)$ and reactance at $5 \mathrm{~Hz}\left(X_{5}\right)$ were measured by impulse oscillometry. We considered $\mathrm{FEF}_{25}-75 \%, \mathrm{FEF} 25-75 \% / \mathrm{FVC}, \mathrm{RV} / \mathrm{TLC}, R 5-R 20$ and $X_{5}$ as small airway measurements.

Thin-slice (i.e. 75-mm) pulmonary CT scans were made at full in- and expiration (RV). PRM was performed to quantify $\mathrm{PRM}^{\mathrm{fSAD}}, \mathrm{PRM}^{\mathrm{Emph}}$ and $\mathrm{PRM}^{\mathrm{PD}}$ as percentage of total lung volume, as described previously [2,3]. We applied linear regression analyses to assess associations between both age and smoking, and $\mathrm{PRM}^{\mathrm{fSAD}}, \mathrm{PRM}^{\mathrm{Emph}}$ and $\mathrm{PRM}^{\mathrm{PD}}$, adjusted for sex. Next, we performed linear regression 
analyses to assess the associations between pulmonary function tests and PRM measurements, adjusted for age, sex, smoking status and height.

CT scans of 49 current smokers and 47 never-smokers were available for analyses; median age was 40 years (interquartile range (IQR) $22-53$ years), $56 \%$ of subjects being males. The mean \pm SD FEV 1 in the study population was $108 \pm 12 \%$ predicted, $\mathrm{FEV}$ / FVC was $80 \pm 6 \%$ and median smoking history among current smokers was 16 pack-years (IQR 4-30 pack-years).

A higher age was significantly associated with more $\mathrm{PRM}^{\mathrm{fSAD}}, \mathrm{PRM}^{\mathrm{Emph}}$ and $\mathrm{PRM}^{\mathrm{PD}}$, independently of smoking and sex (table 1). Current smoking was significantly associated with more $\mathrm{PRM}^{\mathrm{PD}}$, but not with more $\mathrm{PRM}^{\mathrm{fSAD}}$ or $\mathrm{PRM}^{\mathrm{Emph}}$, independently of age and sex.

We investigated whether pulmonary function tests were associated with PRM measurements and found that a lower FEV1/FVC was significantly associated with more $\mathrm{PRM}^{\mathrm{fSAD}}$, independently of age, sex, smoking status and height (table 1). In addition, higher RV/TLC, lower TLCOc/VA and lower FEF25-75\%/FVC were significantly associated with more $\mathrm{PRM}^{\mathrm{fSAD}}$ and $\mathrm{PRM}^{\mathrm{Emph}}$. $R 5-R_{2} 0$ was significantly and negatively associated with $\mathrm{PRM}^{\mathrm{FSAD}}$, but not with $\mathrm{PRM}^{\mathrm{Emph}}$. $\mathrm{PRM}^{\mathrm{PD}}$ was not associated with pulmonary function tests.

We tested whether $\mathrm{PRM}^{\mathrm{fSAD}}$ and $\mathrm{PRM}^{\mathrm{Emph}}$ contributed independently to pulmonary function measurements by including $\mathrm{PRM}^{\mathrm{fSAD}}$ and $\mathrm{PRM}^{\mathrm{Emph}}$ in regression models with FEV1/FVC, FEF25-75\%/FVC, RV/TLC \% predicted, TLC $\%$ predicted, TLCOd $/ V \mathrm{~A} \%$ predicted and $R 5-R 20$, alternately, as outcome parameters. More $\mathrm{PRM}^{\mathrm{fSAD}}$ was significantly associated with lower FEV1/FVC $(\beta=-0.57, \mathrm{p}<0.05)$, lower FEF25-75\%/FVC $(\beta=-0.02, \mathrm{p}<0.01)$ and higher RV/TLC \% predicted $(\beta=1.13, \mathrm{p}<0.05)$, independently of $\mathrm{PRM}^{\mathrm{Emph}}$.

Our study investigated individuals without objective lung disease according to lung function tests and history. The results show that an older age is associated with more extensive small airways disease, as well as more extensive emphysema and parenchymal disease of the lungs, as measured with PRM. In addition, current smokers had more extensive parenchymal disease than never-smokers, independently of age. The more small airway disease and emphysema were present, the higher were RV/TLC values, and the lower TLCOc/VA and FEF25-75\%/FVC values, even in these respiratory healthy subjects. Interestingly, more small airway disease was independent of the extent of emphysema associated with higher RV/TLC \% predicted, lower $\mathrm{FEF}_{25}-75 \% / \mathrm{FVC}$ and lower FEV1/FVC values.

An important finding was the elevated levels of $\mathrm{PRM}^{\mathrm{fSAD}}, \mathrm{PRM}^{\mathrm{Emph}}$ and $\mathrm{PRM} \mathrm{PD}^{\mathrm{PD}}$ with increasing age. Ageing of the lung is related to decreased lung elasticity and increased RV due to collapsibility of the small airways $[7,8]$. We were able to visualise these physiological alterations by using PRM to distinguish between small airway disease, emphysema and parenchymal disease. It has been previously shown that an indirect measurement of small airways disease (i.e. air trapping measured on an expiratory CT scan) increases with

TABLE 1 Linear regression analyses of the association between age, current smoking and pulmonary function tests and parametric response mapping (PRM)

\begin{tabular}{|c|c|c|c|}
\hline & PRM ${ }^{\text {fSAD }}$ & PRM ${ }^{E m p h}$ & PRM $^{\text {PD }}$ \\
\hline Age years ${ }^{\#}$ & $0.06 * *(0.04-0.08)$ & $0.05 * *(0.03-0.06)$ & $0.01 *(0.00-0.01)$ \\
\hline Current smoking" & $-0.14(-0.65-0.37)$ & $-0.42(-0.89-0.05)$ & $0.24 *(0.03-0.44)$ \\
\hline \multicolumn{4}{|l|}{ Pulmonary function tests ${ }^{+}$} \\
\hline $\mathrm{FEV} 1 \mathrm{~L}$ & $0.18(-0.47-0.83)$ & $0.27(-0.33-0.86)$ & $-0.17(-0.43-0.09)$ \\
\hline $\mathrm{FEV}_{1} / \mathrm{FVC} \%$ & $-0.06 * *(-0.12--0.01)$ & $-0.05(-0.10-0.00)$ & $0.01(-0.01-0.03)$ \\
\hline FEF $25-75 \% \mathrm{~L} \cdot \mathrm{S}^{-1}$ & $-0.28(-0.62-0.06)$ & $-0.19(-0.50-0.13)$ & $0.01(-0.13-0.15)$ \\
\hline $\mathrm{FEF}_{25-75 \% / F V C ~ \mathrm{~s}^{-1}}$ & $-2.29 *(-3.84--0.75)$ & $-1.73^{* *}(-3.17--0.29)$ & $0.33(-0.32-0.98)$ \\
\hline RV L & $3.65^{* *}(0.19-7.11)$ & $1.01(-0.40-2.42)$ & $-0.44(-4.72-3.83)$ \\
\hline TLC L & $0.56 * *(0.12-0.99)$ & $0.53 * *(0.13-0.93)$ & $-0.11(-0.29-0.07)$ \\
\hline $\mathrm{RV} / \mathrm{TLC} \%$ & $0.11 *(0.04-0.17)$ & $0.08 * *(0.01-0.14)$ & $0.01(-0.02-0.04)$ \\
\hline$T \mathrm{LCOc} / V_{\mathrm{A}} \mathrm{mmol} \cdot \mathrm{min}^{-1} \cdot \mathrm{kPa}^{-1} \cdot \mathrm{L}^{-1}$ & $-1.97 * *(-3.55--0.39)$ & $-1.62 * *(-3.08--0.16)$ & $0.36(-0.29-1.01)$ \\
\hline$R_{5}-R_{20} \mathrm{kPa} \cdot \mathrm{L}^{-1} \cdot \mathrm{s}^{-1}$ & $-6.59 * *(-12.2--0.92)$ & $-4.84(-10.11-0.43)$ & $0.41(-0.19-2.76)$ \\
\hline & $4.92[-3.51-13.35)$ & $5.02(-2.72-12.76)$ & $-0.62(-4.02$ \\
\hline
\end{tabular}

Data are presented as $\beta$ (95\% CI). PRM values were normalised by natural-logarithmic transformation. $\mathrm{PRM}^{\mathrm{fSAD}}$ : extent of small airway disease; $\mathrm{PRM}^{\mathrm{Emph}}$ : extent of emphysema; $\mathrm{PRM}{ }^{\mathrm{PD}}$ : extent of parenchymal disease; FEV1: forced expiratory volume $1 \mathrm{~s}$; FVC: forced vital capacity; FEF25-75\%: forced expiratory flow at $25-75 \%$ of FVC; RV: residual volume; TLC: total lung capacity; TLCOc: transfer factor of the lung for carbon monoxide correction for haemoglobin; $V_{\mathrm{A}}$ : alveolar volume; $R 5$ : resistance at $5 \mathrm{~Hz}$; $R 20$ : resistance at $20 \mathrm{~Hz}$; $X_{5}$ : reactance at $5 \mathrm{~Hz} .{ }^{*}$ : adjusted for sex and smoking status; " : adjusted for sex and age; ${ }^{+}$: adjusted for age, sex, smoking status and height. Bold indicates statistically significant values. ${ }^{*}: p<0.05 ;{ }^{* *}: p<0.01$. 
age in respiratory-healthy subjects [9]. However, a limitation of such an indirect measurement is that it cannot distinguish air trapping due to emphysema from air trapping due to small airway disease. Furthermore, it is well established that measurements of emphysema on CT scans increase with ageing both in smokers and nonsmokers (never-smokers and ex-smokers), which our findings support [10-12].

We found that current smokers had significantly more $\mathrm{PRM}^{\mathrm{PD}}$ than never-smokers, independently of age. Parenchymal disease is defined as increased parenchymal density upon inspiration and it could be suggested that more $\mathrm{PRM}^{\mathrm{PD}}$ in current smokers reflects an inflammatory process. This hypothesis is supported by a previous study from our group among haematopoietic cell transplant recipients showing that more $\mathrm{PRM}^{\mathrm{PD}}$ is associated with pulmonary infection [3]. No differences in $\mathrm{PRM}^{\mathrm{fSAD}}$ and $\mathrm{PRM}^{\mathrm{Emph}}$ were found between current and never-smokers. This could be due to a lack of sensitivity of PRM or due to the deliberate accrual of smokers with normal pulmonary function. An alternative explanation may be that $\mathrm{PRM}^{\mathrm{PD}}$ "masks" underlying $\mathrm{PRM}^{\mathrm{fSAD}}$ and $\mathrm{PRM}^{\mathrm{Emph}}$ among current smokers.

Finally, more $\mathrm{PRM}^{\mathrm{fSAD}}$ and more $\mathrm{PRM}^{\mathrm{Emph}}$ were found to be associated with higher RV/TLC values and lower TLCOd/VA and FEF25-75\%/FVC values, even in this respiratory-healthy population. This is in line with previous studies reporting that air trapping and emphysema on CT scans correlate with worse pulmonary function [2, 13-15]. To our surprise, we found a higher $R 5-R 20$, i.e. more small airway dysfunction, to be associated with less $\mathrm{PRM}^{\mathrm{fSAD}}$. It is difficult to explain this unexpected finding but it may result from the very small range of $R 5-R 20$ values in our healthy population (IQR $0.00-$ $0.05 \mathrm{kPa} \cdot \mathrm{L}^{-1} \cdot \mathrm{s}^{-1}$ ). Of specific interest is that more $\mathrm{PRM}^{\mathrm{fSAD}}$ was associated with worse pulmonary function independently of $\mathrm{PRM}^{\mathrm{Emph}}$. Since it was previously suggested that small airways disease precedes emphysema [1], we speculate that early changes in pulmonary function are better reflected by $\mathrm{PRM}^{\mathrm{fSAD}}$ than $\mathrm{PRM}^{\mathrm{Emph}}$, suggesting that an increase in PRM ${ }^{\mathrm{fSAD}}$ may be the first sign of pulmonary pathology.

A limitation of the study is the lack of histological samples (i.e. peripheral airway biopsies or lung tissue) for direct comparison with the PRM measurements in order to validate $\mathrm{PRM}^{\mathrm{fSAD}}, \mathrm{PRM}^{\mathrm{Emph}}$ and PRM ${ }^{\mathrm{PD}}$. Furthermore, CT scans are accompanied by radiation exposure, which impedes the application of PRM on a large scale; therefore, future studies are needed to identify subsets of subjects who will benefit from the PRM technique.

In conclusion, our findings show that PRM is a promising tool to characterise early pulmonary alterations in the lungs even without clinical symptomatology, by distinguishing small airway disease, emphysema and parenchymal disease. Future studies are required to assess its role in predicting or phenotyping lung diseases.

- @ERSpublications

Parametric response mapping can distinguish small airway disease, emphysema and parenchymal disease on pulmonary CT http://ow.ly/Neuw6

Ilse M. Boudewijn ${ }^{1,2}$, Dirkje S. Postma ${ }^{1,2}$, Eef D. Telenga ${ }^{1,2}$, Nick H.T. ten Hacken ${ }^{1,2}$, Wim Timens ${ }^{2,3}$, Matthijs Oudkerk', Brian D. Ross ${ }^{5,6}$, Craig J. Galbán ${ }^{5,6,7}$ and Maarten van den Berge e $^{1,2,7}$

${ }^{1}$ Department of Pulmonary Diseases, University of Groningen, University Medical Center Groningen, Groningen, The Netherlands. ${ }^{2}$ University of Groningen, University Medical Center Groningen, Groningen Research Institute for Asthma and COPD, Groningen, The Netherlands. ${ }^{3}$ University of Groningen, University Medical Center Groningen, Department of Pathology and Medical Biology, Groningen, The Netherlands. ${ }^{4}$ University of Groningen, University Medical Center Groningen, Department of Radiology, Groningen, The Netherlands. ${ }^{5}$ Department of Radiology, University of Michigan, Ann Arbor, MI, USA. ${ }^{6}$ Center for Molecular Imaging, University of Michigan, Ann Arbor, MI, USA. ${ }^{7}$ Both authors contributed equally.

Correspondence: Maarten van den Berge, University of Groningen, University Medical Center Groningen, Department of Pulmonary Diseases (AA11), PO Box 30.001, 9700 RB Groningen, The Netherlands. E-mail: m.van.den.berge@umcg.nl

Received: Nov 032014 | Accepted after revision: April 262015 | First published online: June 252015

Support statement: This study was funded by the Royal Netherlands Academy of Arts and Sciences, Stichting Astma Bestrijding Nederland and the US National Institutes of Health (research grant R44HL118837). Funding information for this article has been deposited with FundRef.

Conflict of interest: Disclosures can be found alongside the online version of this article at erj.ersjournals.com

Acknowledgements: The authors thank Jennifer Boes (Department of Radiology and Center for Molecular Imaging, University of Michigan, Ann Arbor, MI, USA) for optimising the registration algorithm used for the PRM analysis.

\section{References}

1 McDonough JE, Yuan R, Suzuki M, et al. Small-airway obstruction and emphysema in chronic obstructive pulmonary disease. N Engl J Med 2011; 365: 1567-1575.

2 Galban CJ, Han MK, Boes JL, et al. Computed tomography-based biomarker provides unique signature for diagnosis of COPD phenotypes and disease progression. Nat Med 2012; 18: 1711-1715. 
Galban CJ, Boes JL, Bule M, et al. Parametric response mapping as an indicator of bronchiolitis obliterans syndrome after hematopoietic stem cell transplantation. Biol Blood Marrow Transplant 2014; 20: 1592-1598.

4 Buist AS, McBurnie MA, Vollmer WM, et al. International variation in the prevalence of COPD (the BOLD Study): a population-based prevalence study. Lancet 2007; 370: 741-750.

5 Miller MR, Hankinson J, Brusasco V, et al. Standardisation of spirometry. Eur Respir J 2005; 26: 319-338.

6 Crapo RO, Casaburi R, Coates AL, et al. Guidelines for methacholine and exercise challenge testing - 1999. This official statement of the American Thoracic Society was adopted by the ATS Board of Directors, July 1999. Am J Respir Crit Care Med 2000; 161: 309-329.

7 Dyer C. The interaction of ageing and lung disease. Chron Respir Dis 2012; 9: 63-67.

8 Janssens JP, Pache JC, Nicod LP. Physiological changes in respiratory function associated with ageing. Eur Respir J 1999; 13: 197-205.

9 Lee KW, Chung SY, Yang I, et al. Correlation of aging and smoking with air-trapping at thin-section CT of the lung in asymptomatic subjects. Radiology 2000; 214: 831-836.

10 Gevenois PA, Scillia P, de Maertelaer V, et al. The effects of age, sex, lung size, and hyperinflation on CT lung densitometry. AJR Am J Roentgenol 1996; 167: 1169-1173.

11 Wang Q, Takashima S, Wang JC, et al. Prevalence of emphysema in individuals who underwent screening CT for lung cancer in Nagano prefecture of Japan. Respiration 2001; 68: 352-356.

12 Camiciottoli G, Cavigli E, Grassi L, et al. Prevalence and correlates of pulmonary emphysema in smokers and former smokers. A densitometric study of participants in the ITALUNG trial. Eur Radiol 2009; 19 : 58-66.

13 Matsuoka S, Kurihara Y, Yagihashi K, et al. Quantitative assessment of air-trapping in chronic obstructive pulmonary disease using inspiratory and expiratory volumetric MDCT. AJR Am J Roentgenol 2008; 190: 762-769.

14 Bommart S, Marin G, Bourdin A, et al. Relationship between CT air-trapping criteria and lung function in small airway impairment quantification. BMC Pulm Med 2014; 14: 29.

15 Schroeder JD, McKenzie AS, Zach JA, et al. Relationships between airflow obstruction and quantitative CT measurements of emphysema, air-trapping, and airways in subjects with and without chronic obstructive pulmonary disease. AJR Am J Roentgenol 2013; 201: W460-W470.

\section{Accuracy of chest high-resolution computed tomography in diagnosing diffuse cystic lung diseases}

To the Editor:

The diffuse cystic lung diseases (DCLDs) are a group of pathophysiologically heterogeneous processes characterised by the presence of multiple, thin-walled, air-filled spaces within the pulmonary parenchyma [1]. The differential diagnosis of DCLDs includes lymphangioleiomyomatosis (LAM), follicular bronchiolitis (FB), lymphocytic interstitial pneumonia (LIP), Birt-Hogg-Dubé syndrome (BHD), pulmonary Langerhans cell histiocytosis (PLCH), amyloidosis, light chain deposition disease, cystic metastases, infectious entities such as Pneumocystis, and other aetiologies [2]. Bronchiectasis and bullous changes seen in chronic obstructive pulmonary disease can also produce high-resolution computed tomography (HRCT) patterns that mimic the DCLDs.

The utility of HRCT in the diagnosis of LAM and differentiation from other DCLDs is not completely defined. According to the European Respiratory Society (ERS) guidelines, characteristic HRCT features along with a compatible clinical history are sufficient to confidently diagnose LAM, without the need for a tissue biopsy [3]. However, previously reported accuracy rates for diagnosing LAM based on HRCT findings may not be sufficient in an era when interventions with substantial risks are becoming available. Two prior studies have reported accuracy rates of $72-84 \%$ in diagnosing LAM based on imaging characteristics alone $[4,5]$. The aim of our study was to determine the diagnostic accuracy of HRCT evaluation by radiologists and pulmonologists, at various levels of expertise, in patients with DCLDs presenting to referral centres.

We retrospectively obtained HRCTs from 89 patients referred to LAM Foundation Clinics at the University of Cincinnati (Cincinnati, OH, USA), Mayo Clinic Rochester (Rochester, MN, USA) and National Kinki-Chou Hospital (Osaka, Japan) for further evaluation of DCLDs. All scans were non-contrast HRCTs and only thin section (1-3 mm) images were employed in the analysis. Patient 